Copyright by the American Institute of Physics (AIP). Dong, SX; Li, JF; Viehland, D; et al., "A strong magnetoelectric voltage gain effect in magnetostrictive-piezoelectric composite," Appl. Phys. Lett. 85, 3534 (2004); http:// dx.doi.org/10.1063/1.1786631

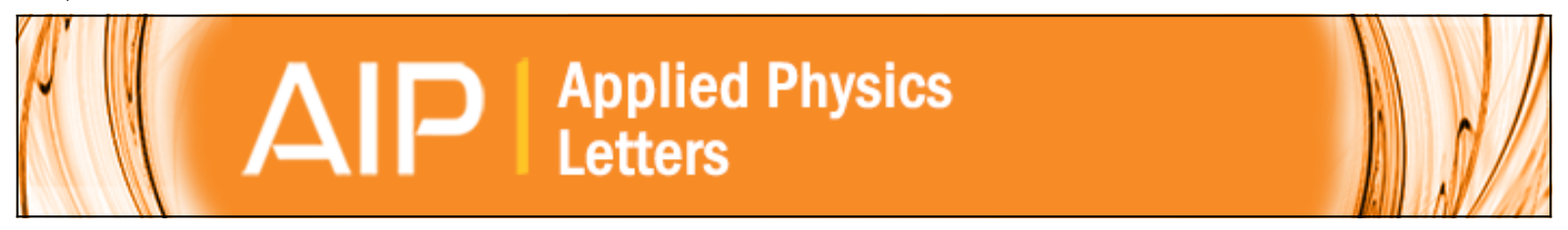

\title{
A strong magnetoelectric voltage gain effect in magnetostrictive-piezoelectric composite
}

Shuxiang Dong, J. F. Li, D. Viehland, J. Cheng, and L. E. Cross

Citation: Applied Physics Letters 85, 3534 (2004); doi: 10.1063/1.1786631

View online: http://dx.doi.org/10.1063/1.1786631

View Table of Contents: http://scitation.aip.org/content/aip/journal/apl/85/16?ver=pdfcov

Published by the AIP Publishing

\section{Over 700 papers \& presentations on multiphysics simulation visw now

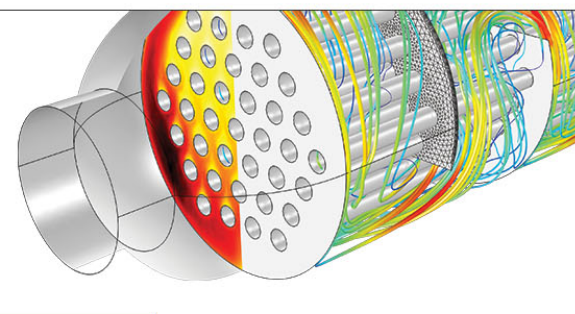




\title{
A strong magnetoelectric voltage gain effect in magnetostrictive- piezoelectric composite
}

\author{
Shuxiang Dong, ${ }^{\text {a) }}$ J. F. Li, and D. Viehland \\ Materials Science \& Engineering, Virginia Tech, Blacksburg, Virginia 24061 \\ J. Cheng and L. E. Cross \\ Materials Research Laboratory, Pennsylvania State University, State College, Pennsylvania 16802
}

(Received 9 April 2004; accepted 6 July 2004)

\begin{abstract}
A magnetoelectric laminate composite consisting of magnetostrictive Terfenol-D $\left(\mathrm{Tb}_{1-x} \mathrm{Dy}_{x} \mathrm{Fe}_{2-y}\right)$ and piezoelectric $\mathrm{Pb}(\mathrm{Zr}, \mathrm{Ti}) \mathrm{O}_{3}$ layers has an extremely high voltage gain effect of $\approx 300$ at its resonant state, offering potential for high-voltage miniature transformer applications. () 2004 American Institute of Physics. [DOI: 10.1063/1.1786631]
\end{abstract}

The magnetoelectric (ME) effect is a polarization response to an applied magnetic field $H$, or conversely a spin response to an applied electric field $E$. ${ }^{1}$ Ferroelectromagnetic materials of single phase, multiple phases, and laminate composites have been studied. ${ }^{2-15}$ However, to date, investigations of ME laminate composites have focused on passive sensors, ${ }^{6,12,14}$ rather than high-power device applications. Also, previously, only ME materials with low coupling have been found.

It is possible that ME laminates could operate under high-power drive in solid-state transformers. This is because both magnetostrictive Terfenol-D $\left(\mathrm{Tb}_{1-x} \mathrm{Dy}_{x} \mathrm{Fe}_{2-y}\right)$ and piezoelectric $\mathrm{Pb}(\mathrm{Zr}, \mathrm{Ti}) \mathrm{O}_{3} \quad$ (PZT) layers exhibit significant magneto-mechanical and electro-mechanical energy densities, respectively. ${ }^{16-18}$ Recently, we have developed laminate composite designs that have significantly higher magnetoelectric coupling effects. ${ }^{13,14}$ Our approach was based on energy analysis and laws of motion, and not simply constitutive equations. ${ }^{9,11}$ The analysis was developed for a long plate type piezoelectric/magnetostrictive laminate composite; and was based upon the piezoelectric and piezomagnetic equations of state, in a longitudinal-mode vibration. Our analysis revealed the possibility of an extremely high magnetoelectric voltage gain, suitable for high-voltage miniature transformer applications.

Figure 1 illustrates the composite geometry chosen for this investigation. It is a long plate type piezoelectric/ magnetostrictive laminate composite, in which the piezoelectric layer is sandwiched between two magnetostrictive ones. More complicated multilayer geometries of this general type are possible, but that given in Fig. 1 readily allows for equivalent circuit analysis. The conductive magnetostrictive layers are separated by an insulating piezoelectric one, and thus eddy currents are effectively eliminated if the thickness of the magnetostrictive layers is sufficiently thin. This magnetoelectric laminate design differs from previous ones, as its aspect ratio is high, favoring the longitudinal direction along which fields are applied. The piezoelectric layer consists of two elements, both of which are longitudinally poled and placed in reverse directions with respect to each other about the mid-section of the laminate. This maximizes the voltage and power outputs of the device.
The working principle is as follows. A harmonic ac magnetic field $H_{\mathrm{ac}}$ is applied along the longitudinal direction of the composite. This causes the two magnetostrictive layers to shrink/expand in response to $H_{\mathrm{ac}}$. The magnetostrictive strain acts upon the piezoelectric layer that is bonded between the two magnetostrictive layers, causing the piezoelectric layer to strain, producing a voltage output between the end and middle electrodes. This transduction of magnetic to electrical energy is what we designate as the magnetoelectric coupling effect.

A solenoid with $N$ turns around the laminate that carries a current of $I_{\text {in }}$ was used to excite an ac magnetic field $H_{\text {ac }}$, as shown in Fig. 1. The input ac voltage applied to the coils was $V_{\text {in }}$, and its frequency was $f$. This excites an $H_{\text {ac }}$ of the same frequency $f$, along the longitudinal direction of the laminate. When the frequency of $H_{\mathrm{ac}}$ is equal to the resonance frequency $\left(\omega_{s}=2 \pi f_{s}\right)$ of the laminate, the magnetoelectric coupling effect is sufficiently strong that the output ME voltage $\left(V_{\text {out }}\right)$ induced in the piezoelectric layer is much higher than $V_{\text {in }}$, in particular when a suitable dc magnetic bias $H_{\mathrm{dc}}$ is applied. Thus, under resonant drive, our ME laminate exhibits a strong voltage gain, due to the magnetoelectric effect.

At the first longitudinal resonance frequency, this laminate is a half-wavelength $(\lambda / 2) \mathrm{ME}$ resonator. A node line is located at the middle position of the laminate, where the vibration velocity (i.e., mechanical current) is zero. Assuming a symmetric vibration of the laminate and that the polarization of the piezoelectric layer is symmetric about the node line, the ME voltage induced across each end and middle electrode in the layer is equivalent. The magneto-elastoelectric equivalent circuits can be derived by using the piezoelectric and piezomagnetic constitutive equations, and by applying Newton's second law of motion to the laminate and

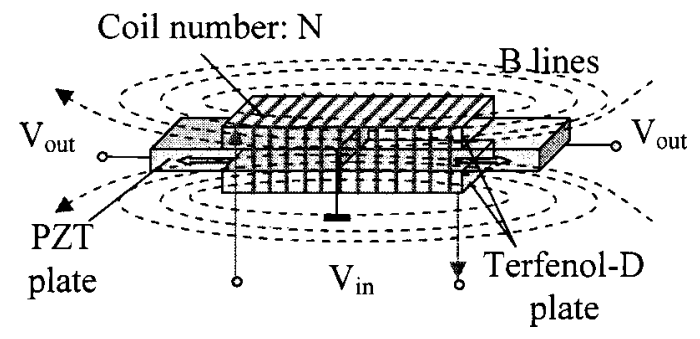

FIG. 1. Configuration and operation principle of magnetoelectric transformer. 


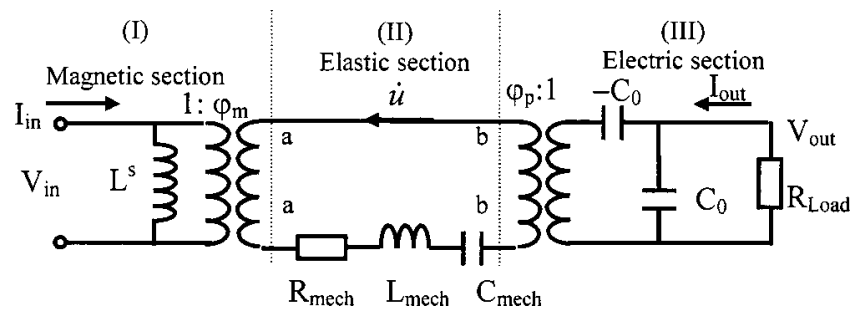

FIG. 2. Equivalent circuit of the ME transformer, where $R_{m}$ $=\pi Z_{0} / 4 Q_{m}, L_{m}=\pi Z_{0} / 4 \omega_{s}$, and $C_{m}=1 / \omega_{s}^{2} L_{m}$ are the motional mechanical impedance, inductance, and capacitance, respectively; $\varphi_{m}=\lambda_{33} / j \omega N$ is the magneto-elastic coupling factor; $\varphi_{p}=2 A_{1} g_{33 p} / l s_{33}^{D} \bar{\beta}_{33}$ is the elasto-electric coupling factor; $C_{0}=2 A_{1} / l \bar{\beta}_{33}$ is the clamped capacitance of the piezoelectric layer; and $L^{s}=2 A_{m} \mu_{33}^{s} N^{2} / l$ is the clamped inductance of the coils around Terfenol-D layers.

subsequently finding analogous electrical parameters. ${ }^{16,17}$ See Fig. 2.

The magnetoelectric voltage gain was determined by analysis of the equivalent circuit in Fig. 2. Assuming that the circuit is unloaded and by applying Ohm's law, the maximum voltage gain can be estimated as

$$
V_{\text {gain, } \max }=\frac{4 Q_{\operatorname{mech}} \varphi_{p}^{2}}{\pi \omega_{s} C_{0} Z_{0}}
$$

where $Q_{\text {mech }}$ is the mechanical quality factor of the piezoelectric layer, $\varphi_{p}$ is the elasto-electric coupling factor, $C_{0}$ is the clamped capacitance of piezoelectric layer, and $Z_{0}$ is the mechanical impedance of the laminate. From this relationship, it can be seen that the maximum voltage gain at the resonance frequency is mainly related to the piezoelectric section of the equivalent circuit in Fig. 2. The voltage gain is directly proportional to $Q_{\text {mech }}$ and $\varphi_{p}^{2}$ (or $g_{33, p}^{2}$, piezoelectric voltage constant) in the piezoelectric layer. This is because the output voltage $V_{\text {out }}$ is generated by this section. The function of the magnetic section of the circuit is to transduce the magnetic energy into a mechanical vibration. The piezoelectric one subsequently transduces this vibration to an electrical output.

Calculations were performed using Eq. (1), assuming a laminate length of $70 \mathrm{~mm}$, width of $10 \mathrm{~mm}$, and thickness of $6 \mathrm{~mm}$. The voltage gain for a value of $Q_{m}=100$ was only 18.5. However, for $Q_{\text {mech }}=500$, the gain was 92.5 . A typical value of $Q_{\text {mech }}$ for PZT-8 is 1400 (see Table I): using this value, a maximum voltage gain of 259 can be estimated. This voltage gain is significantly larger than that of other voltage gain devices, such as electromagnetic and piezoelectric transformers. ${ }^{19-21}$ Thus, the high voltage gain of our laminate could be quite purposeful for power electronics, such as transformer applications.

We can estimate the maximum efficiency $\left(\eta_{\max }\right)$ of the magnetoelectric transformer, using the equivalent circuit in Fig. 2. To do this, we neglect the electrical losses (mainly eddy current loss in magnetoelectric material) by assuming

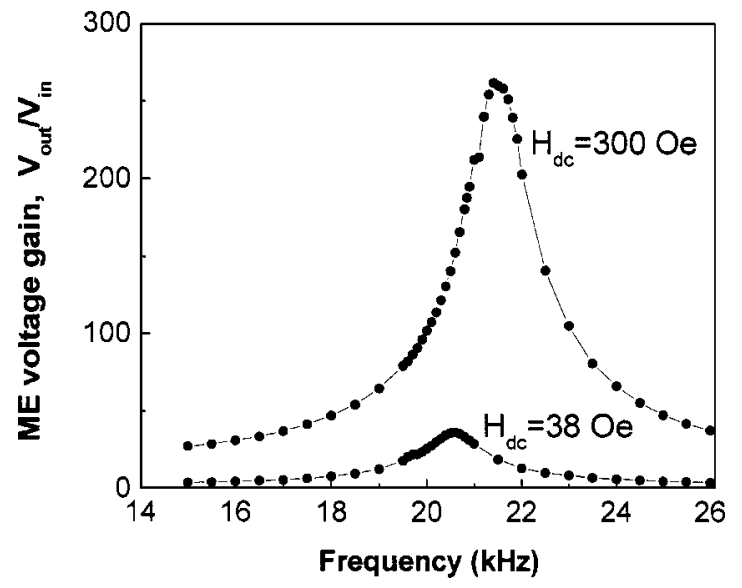

FIG. 3. Measured ME voltage gain as a function of operational frequency.

that the laminate (i) is a multi-thin-layer type; (ii) is operated at relative low-frequency; and (iii) has only mechanical contributions to the total loss factor. Thus, if the load of the circuit is optimum, $R_{\text {load,opt }}=1 / \omega_{s} C_{0}$, the value of $\eta_{\max }$ of the laminate at resonance is

$$
\eta_{\max }=\frac{\varphi_{p}^{2}}{\varphi_{p}^{2}+\frac{\pi Z_{0} C_{0} \omega_{s}}{2 Q_{\text {mech }}}} .
$$

Clearly, a higher $Q_{\text {mech }}$ will result in higher efficiencies. Using the material parameters shown in Table I and by assuming that $Q_{\text {mech }}=1000$, the maximum efficiency of our ME transformer can be estimated using Eq. (2) as $\eta_{\max }>95 \%$ when the eddy current loss in magnetostrictive material can be neglected. When eddy current losses cannot be neglected, the parameters in the magnetic and elastic section will need to be complex coefficients. Correspondingly, the efficiency shall be less than $\eta_{\max }$, and Eq. (2) will need to be modified further.

A laminate composite consisting of a longitudinally poled piezoelectric $\mathrm{Pb}(\mathrm{Zr}, \mathrm{Ti}) \mathrm{O}_{3}(\mathrm{PZT})$ layer and two longitudinally magnetized magnetostrictive Terfenol-D ones was fabricated as shown in Fig. 1. In our design, the Terfenol-D layers were $40 \mathrm{~mm}$ in length, and the piezoelectric layer was $80 \mathrm{~mm}$ in length. A long and thin piezoelectric layer helps to achieve higher ME voltages, as it has larger vibration amplitudes at both ends. The ME voltage gain was measured. A voltage generator was used as an input source to the coils, and an oscilloscope was used for monitoring both input and output voltages. Figure 3 shows the measured voltage gain $V_{\text {out }} / V_{\text {in }}$ of our ME transformer as a function of the drive frequency $f$. A maximum voltage gain of $\sim 260$ was found at a resonance frequency of $21.3 \mathrm{kHz}$. In addition, at the resonance state, the maximum voltage gain of the ME transformer was strongly dependent on an applied dc magnetic bias $H_{\mathrm{dc}}$, which is due to the fact that Terfenol-D has a large

\begin{tabular}{|c|c|c|c|c|c|c|c|c|}
\hline & $g_{33} \times 10^{-3}$ & $d_{33, m}$ or $d_{33, p}$ & $d_{31, m}$ or $d_{31, p}$ & $S_{11}^{H}$ or $S_{11}^{E} \times 10^{-12} \mathrm{~m}^{2} / \mathrm{N}$ & $S_{33}^{H}$ or $S_{33}^{F} \times 10^{-12} \mathrm{~m}^{2} / \mathrm{N}$ & $k_{33}$ & $k_{31}$ & $Q_{m}$ \\
\hline Terfenol-D ${ }^{\mathrm{a}}$ & & $1.1 \times 10^{-8} \mathrm{~Wb} / \mathrm{N}$ & $-5.8 \times 10^{-9} \mathrm{~Wb} / \mathrm{N}$ & 125 & 40 & 0.7 & & $<10$ \\
\hline PZT- $8^{\mathrm{b}}$ piezoceramic & 25.5 & $290 \mathrm{pC} / \mathrm{N}$ & $-125 \mathrm{pC} / \mathrm{N}$ & 11.8 & 17.4 & 0.68 & 0.33 & 1400 \\
\hline
\end{tabular}

TABLE I. Electromechanical and magnetoelastic materials parameters for Terfenol-D and PZT ceramics. 
effective piezomagnetic coefficient only under a suitable $H_{\mathrm{dc}}$. For $H_{\mathrm{dc}} \approx 300 \mathrm{Oe}$, our prototype exhibited a maximum voltage gain of $\approx 300$, which is slightly higher than the predicted value using Eq. (1). It is because the piezoelectric layer is longer than the Terfenol-D layers. Because the effective operational working frequency (assuming neglible eddy currents) for a bulk Terfenol-D is less than $10 \mathrm{kHz},{ }^{17}$ the efficiency of a magnetoelectric transformer consisting of a Terfenol-D/PZT bulk laminate is much lower $\left(\leqslant 0.5 \eta_{\max }\right)$ than that predicted by Eq. (2). However, by using a multithin-layer type configuration, we believe that it may be possible to obtain a higher efficiency, as predicted by Eq. (2).

Compared with conventional electromagnetic transformers, our ME transformer does not require secondary coils with a high-turns ratio in order to obtain a step-up voltage output. Compared with piezoelectric transformers, it has significantly higher voltage gains and a notably wider bandwidth. Also, it has the additional advantage of low input impedance, thus low-voltage current driving for the magnetostrictive Terfenol-D layers, and a high output impedance for the PZT one. Finally, Terfenol-D has a very high energy density ${ }^{17}$ of $4.9-25 \mathrm{~kJ}-\mathrm{m}^{3}$, which is notably higher than that of PZT used in conventional piezoelectric transformers. The combination of these advantages offers potential for applications in new solid-state transformer devices.

In summary, a strong magneto-electric voltage gain effect has been found in laminate composites of piezoelectric PZT and magnetostrictive Terfenol-D. We believe these results have important ramifications, potentially offering applications in miniature solid-state power transformers.

The authors gratefully acknowledge the support of the Office of Naval Research under Grant Nos. N000140210340, N000140210126, and MURI N000140110761.
${ }^{1}$ L. D. Landau and E. Lifshitz, Electrodynamics of Continuous Media (Pergamon, Oxford, 1960), p. 119.

${ }^{2}$ M. Avellaneda and G. Harshe, J. Intell. Mater. Syst. Struct. 5, 501 (1994).

${ }^{3}$ T. Wu and J. Huang, Int. J. Solids Struct. 37, 2981 (2000).

${ }^{4}$ K. Mori and M. Wuttig, Appl. Phys. Lett. 81, 100 (2002).

${ }^{5}$ J. Ryu, A. V. Carazo, K. Uchino, and H. Kim, J. Electroceram. 7, 24 (2001).

${ }^{6}$ J. Ryu, A. V. Carazo, K. Uchino, and H. Kim, Jpn. J. Appl. Phys., Part 1 40, 4948 (2001).

${ }^{7}$ J. Ryu, S. Priya, K. Uchino, H. Kim, and D. Viehland, J. Korean Ceramic Soc. 39, 813 (2002).

${ }^{8}$ C.-W. Nan, M. Li, and J. H. Huang, Phys. Rev. B 63, 144415 (2001).

${ }^{9}$ C. W. Nan, L. Liu, N. Cai, J. Zhai, Y. Ye, and Y. H. Lin, Appl. Phys. Lett. 813831 (2002).

${ }^{10}$ M. Avellaneda and G. Harsche, J. Intell. Mater. Syst. Struct. 5, 501 (1994).

${ }^{11}$ G. Srinivasan, E. Rasmussen, B. Levin, and R. Hayes, Phys. Rev. B 65, 134402 (2002)

${ }^{12}$ Y. Li, Vol. 1 (sensors online: http:/sensorsmag.com/articles/1000/52/ index.htm)

${ }^{13}$ S. Dong, F. Bai, J. F. Li, and D. Viehland, IEEE Trans. Ultrason. Ferroelectr. Freq. Control 50, 1236 (2003).

${ }^{14}$ S. Dong, J. F. Li, and D. Viehland, Appl. Phys. Lett. 83, 11 (2003).

${ }^{15}$ G. Srinivasan, E. T. Rasmussen, J. Gallegos, R. Srinivasan, Y. I. Bokhan, and V. M. Laletin, Phys. Rev. B 64, 214408 (2001).

${ }^{16} \mathrm{~W}$. Mason, Physical Acoustics, Principles and Methods (Academic, New York, 1964), Part 1A.

${ }^{17}$ G. Engdahl, Magnetostrictive Materials Handbook (Academic, San Diego, 2000).

${ }^{18}$ T. Ikeda, Fundamentals of Piezoelectricity (Oxford University Press, New York, 1990).

${ }^{19}$ C. Rosen, Seventh Electronic Components Symposium, Washington, DC, 1956, pp. 205.

${ }^{20}$ J. S. Yang and W. Zhang, Int. J. Appl. Electromagn. Mech. 10, 105 (1999).

${ }^{21}$ J. S. Yang, X. Zhang, Int. J. Appl. Electromagn. Mech. 16, 29 (2002). 